European Psychiatry

www.cambridge.org/epa

\section{Research Article}

Cite this article: Buchanan A, Moore KE, Pittman B, McKee SA (2021). Psychosocial function, legal involvement and violence in mental disorder. European Psychiatry, 64(1), e75, 1-8

https://doi.org/10.1192/j.eurpsy.2021.2250

Received: 06 August 2021

Revised: 29 October 2021

Accepted: 01 November 2021

\section{Keywords:}

Diagnosis; violent behavior; psychosocial function; incarceration; arrest

Author for correspondence:

*Alec Buchanan,

E-mail: alec.buchanan@yale.edu

\title{
Psychosocial function, legal involvement and violence in mental disorder
}

\section{Alec Buchanan $^{1,2 *}$ (D), Kelly E. Moore ${ }^{3}$ (D), Brian Pittman ${ }^{4}$ and Sherry A. McKee ${ }^{4}$}

${ }^{1}$ Law and Psychiatry Division, Yale School of Medicine, New Haven, Connecticut 06519, USA; ${ }^{2}$ VA Connecticut Health Care System, West Haven, Connecticut 06516, USA; ${ }^{3}$ Department of Psychology, East Tennessee State University, Johnson City, Tennessee 37614, USA and ${ }^{4}$ Department of Psychiatry, Yale School of Medicine, New Haven, Connecticut 06519, USA

\section{Abstract}

Background. The correlates of legally significant outcomes that have been identified in people with mental disorders are of limited value in understanding the mechanisms by which these outcomes occur.

Aims. To describe the relationships between mental disorder, impaired psychosocial function, and three legally significant outcomes in a representative sample of the US population.

Methods. We used a population survey, the National Epidemiologic Survey on Alcohol and Related Conditions (NESARC-III, sample size 36,309), to identify people who self-reported serious trouble with the police or the law over the past 12 months and two lifetime outcomes, being incarcerated and engaging in violence to others. DSM-5 categories were generated using the Alcohol Use Disorder and Associated Disabilities Interview Schedule-5. Psychosocial function was assessed using social and role-emotional function scores of the 12-Item ShortForm Health Survey Version 2.

Results. Participants with mental disorder, but not people with no diagnosis, who reported serious trouble with the police or with the law during the previous 12 months reported significantly worse psychosocial function than those who did not report such trouble. The size of the statistical effect varied by diagnosis, moderate for some forms of mental illness and for alcohol abuse and nonsignificant for drug abuse and the personality disorders. Effect sizes were largest for diagnoses where legally significant outcomes were least common.

Conclusions. The effect of impaired psychosocial function, for instance in disrupting family and social networks that would otherwise protect against these legally significant outcomes, warrants further investigation in studies with longitudinal designs.

\section{Introduction}

The relationship between mental disorder and legally significant outcomes such as legal trouble, incarceration, and violence are clinically important but empirically unresolved. Bivariate analyses of population surveys and cohort samples most frequently focus on violence and report rates that are three to four times higher than in the general population for depression, anxiety disorder, and eating disorder [1] and for categories that combine diagnoses such as major mental disorder [2]. Rates are of violence are 10 times higher for substance use disorder [3] and two to three times higher again for people with multiple psychiatric diagnoses [4]. Sociodemographic characteristics and past behavior are also known correlates of legally significant outcomes and the statistical effects of all forms of mental disorder are smaller when multivariate approaches are used to control for these variables $[5,6]$.

Only in psychosis, however, have these findings been followed by significant progress in identifying mechanisms that may explain the association between mental disorder, on the one hand, and legal trouble, incarceration and violence, on the other [7]. In particular, the relative contributions of symptoms and signs of illness, of labeling and stigma and of known risk factors that are unrelated to mental state, remain largely unknown. Given the large number of diagnoses involved, one way forward is to identify signs and symptoms of mental disorder that can be used across diagnoses to identify the times and circumstances in which legal involvement is most likely, to suggest explanations and, hence, to inform interventions.

One candidate for signs and symptoms that may offer clues as to mechanism is the impairment of psychosocial function that all mental disorders cause [8]. Functional impairment is a recognized marker of illness severity and a criterion for diagnosis in each of the recognized international classifications of mental disorder $[9,10]$. However, the relationship between diagnosis, function and legal involvement has been studied only infrequently and in treatment samples, where selection bias limits the generalizability of findings $[11,12]$. The limited data that do exist suggest that for some diagnoses function exerts an interactive effect, protecting against violent behavior in people with psychosis who have frequent social contacts [13], or a 
"curvilinear" effect, whereby violent behavior is less frequent at extremes of social function and more frequent when function is moderate [14].

We used the third wave of a national community survey sample, the National Epidemiologic Survey on Alcohol and Related Conditions (NESARC-III), to test whether people with mental disorders who describe serious trouble with the law or the police, being incarcerated or engaging in violence also describe impaired psychosocial function.

\section{Method}

\section{Sample and procedures}

The NESARC-III is a cross sectional representative survey of the civilian US population sponsored by the National Institute on Alcohol Abuse and Alcoholism (NIAAA). The survey conducted in-person interviews with noninstitutionalized US adults, including the residents of group and rest homes, between April of 2012 and June of 2013. The final sample size was 36,309. The person-level response rate was $84 \%$. Consent procedures were approved by the National Institutes of Health. Detailed descriptions of the methodology have been published $[15,16]$.

\section{Variables}

Diagnoses for mood disorders, anxiety disorders, PTSD, eating disorders, substance use disorders, and personality disorders were generated using the Alcohol Use Disorder and Associated Disabilities Interview Schedule (AUDADIS)-5, a structured interview generating DSM categories. Test-retest reliability for the AUDADIS-5 ranges from fair to excellent and is similar to that for other structured diagnostic interviews [17]. Personality disorder diagnoses were generated for a participant's lifetime. Other diagnoses were generated for two time periods, past year and lifetime. We used the response on two NESARC-III items, "In the last 12 months, did a doctor or other health professional tell you that you had schizophrenia or a psychotic illness or episode?" and, "Did this happen before 12 months ago?" to generate two further variables, past year and lifetime "schizophrenia/psychosis."

We created three binary outcome variables relating to behavioral outcomes. One used the NESARC-III item, "Please tell me if you have had any of the following experiences in the last 12 months ... did you have serious trouble with the police or the law?" The second measured lifetime incarceration using the NESARC-III item, "Since you were 18, were you ever in jail, prison or a correctional facility?" The third measured a lifetime history of violence using, as elsewhere [18], a positive response to any of 7 NESARC-III items relating to violent behavior occurring since the age of 15: stole directly (by mugging or making threats with a weapon); forced sexual activity; got into fights one started; physically hurt someone; exchanged blows in a fight; used a weapon; and hit someone causing injury or hospitalization.

We measured psychosocial function using the 12-Item ShortForm Health Survey Version 2 (SF-12v2) [19] which generates norm-based disability scores with a mean (SD) of 50 (10) and a range of $0-100$. Lower scores indicate greater disability. Social function is defined by the SF-12v2 as the extent to which a subject perceives mental health problems as having interfered with their normal social activities $[20,21]$. One item measuring social functioning asks, "During the past 4 weeks, how much of the time have your physical health or emotional problems interfered with your social activities like visiting with friends, relatives, and so forth."

Role-emotional functioning in the SF-12v2 addresses the extent to which a subject's symptoms lead them to cut down the amount of time spent on work, to accomplish less than they would like at work and to carry out daily activities less carefully than usual. Items include, "During the past 4 weeks, tell me how much of the time you have had any of the following problems with your work or other regular daily activities as the result of any emotional problems, such as feeling depressed or anxious," "How much of the time have you accomplished less than you would like?" and, "How much of the time have you not done work or other activities as carefully as usual?"

Testing shows the SF-12v2 to be a reliable and valid measure of function [19]. We did not include two scores that it can be used to generate, mental health and the mental component summary, because of the similarity of some of their items (feeling "nervous" or "downhearted and blue") to AUDADIS-5 diagnostic criteria. We included only those personality disorder diagnoses for which AUDADIS- 5 reliability data have been published: borderline, antisocial and schizotypal [17].

\section{Statistical analyses}

We analyzed data on all subjects from NESARC-III for whom data on level of function were available $(n=36,293)$ using the logistic and linear regression survey procedures in SAS, version 9.4 [22]. These procedures allowed us to incorporate the stratification, clustering, and unequal weighting of the study sampling design and enabled the results to reflect three sociodemographic characteristics (age, sex, and self-defined ethnicity) of the US population.

We calculated rates for each outcome for each diagnosis. We used 12-month diagnoses to calculate rates for the 12-month outcome (serious trouble with the police or the law) and lifetime diagnoses to calculate rates for lifetime outcomes (incarceration and violence others). Rates were compared using logistic regression to generate odds ratios (ORs). We calculated mean social functioning and role-emotional functioning scores for subjects who did and who did not meet criteria for each outcome variable. The differences between these means were compared using linear regression. We report statistical significance and effect size. We describe effect size using Cohen's $d$. We refer to effect sizes of between 0.2 and 0.5 as "small" and those of 0.5 and over as "moderate."

\section{Results}

The sample is described in Table 1. A total of 8,532 participants (23.5\%) received a diagnosis of mental illness for the previous 12 months. Of these, $3.4 \%$ had encountered serious trouble with the police or the law compared with only $0.7 \%$ of participants with no 12 -month diagnosis (OR of 5.6; 95\% CI 4.6-6.9). The equivalent ORs for any substance abuse diagnosis and any personality disorder were 10.5 (95\% CI 8.6-12.7) and 10.6 (95\% CI 8.3-13.5), respectively. Among individual diagnoses, ORs were highest for drug abuse (OR 19.9; 95\% CI 15.5-25.5), schizophrenia/psychosis (17.5; 95\% CI 10.6-28.9) and antisocial personality disorder (16.6; 95\% CI 12.2-22.6) and lowest for eating disorders (2.3; 95\% CI 1.0-5.6) and specific phobias (4.3; 95\% CI 3.0-6.2).

A total of 12,296 participants (33.9\%) received a lifetime mental illness diagnosis. Of these, $15.8 \%$ had been incarcerated and $35.9 \%$ had committed one or more act of violence. Compared to people 
Table 1. Description of sample: diagnosis and outcomes variables $(n=36,293)$.

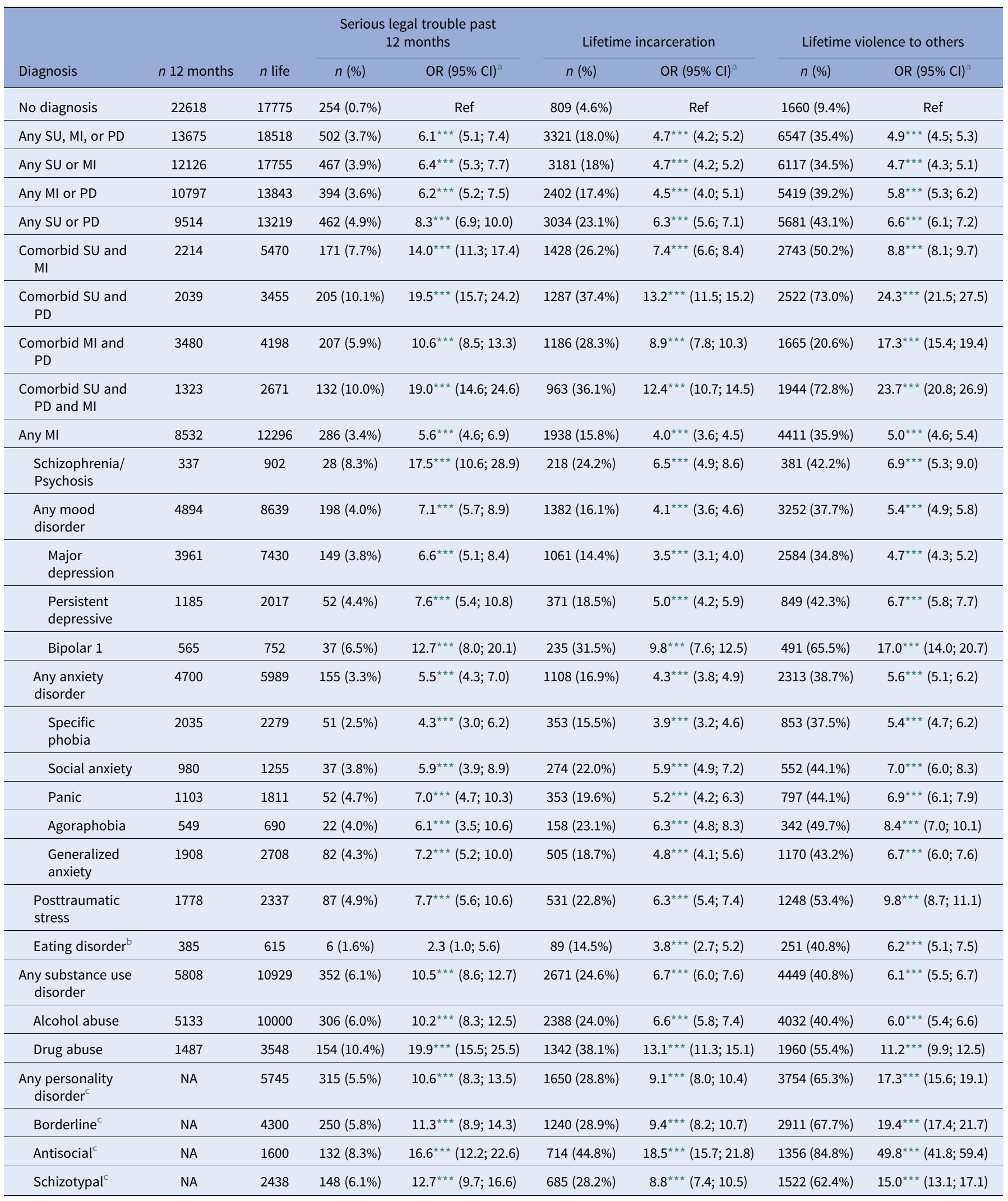

Note: Sample is limited to people with data on functional impairment. Psychiatric categories are not mutually exclusive. Lower scores indicate poorer perceived functioning.

${ }^{\star \star \star} p<0.001$.

${ }^{a}$ Data weighted to adjust for nonresponse.

${ }^{b}$ Includes bulimia and anorexia nervosa.

conly lifetime personality disorder diagnoses are available. 
with no diagnosis, legal involvement was again commoner in people with mental illness (lifetime incarceration OR 4.0 [95\% CI 4.6-6.9]; lifetime violence OR 5.0 [95\% CI 4.6-6.9]), substance abuse (lifetime incarceration OR 6.7 (95\% CI 6.0-7.6); lifetime violence OR 6.1 (95\% CI 5.5-6.7); and personality disorders (lifetime incarceration OR 9.1 [95\% CI 8.0-01.4]; lifetime violence OR 17.3 [95\% CI 15.6-19.1]). For both 12-month and lifetime measures, having comorbid diagnoses from multiple categories (mental illness, substance abuse, and personality) was associated with a higher OR for all three outcomes.

The association, broken down by diagnosis, between having encountered serious trouble with the police or the law over the past 12 months and impaired psychosocial function is shown in
Table 2. Participants with a mental disorder who reported such trouble described lower levels of function than those who did not report such trouble. For participants without a mental disorder there was no effect. For specific phobia (0.57), social anxiety (0.52), agoraphobia (0.52), and eating disorder (0.60) effect sizes were moderate. For other forms of mental illness effect sizes were small (0.32-0.49). For alcohol abuse the effect size of social functioning was again small (0.34) and for drug abuse and personality disorders it was not significant. Role-emotional function showed a similar pattern to social function but with smaller effect sizes.

The pattern was similar, also with smaller effect sizes, for the lifetime outcome variables: incarceration and violence to others

Table 2. Serious legal trouble (SLT) past 12 months: effect size of functional impairment by 12-month diagnosis.

\begin{tabular}{|c|c|c|c|c|c|c|c|c|}
\hline \multirow[b]{2}{*}{ Diagnosis past 12 months } & \multicolumn{4}{|c|}{ Social functioning } & \multicolumn{4}{|c|}{ Role-emotional functioning } \\
\hline & $\begin{array}{l}\text { SLT present Mean } \\
\text { (SE) }\end{array}$ & $\begin{array}{c}\text { SLT absent Mean } \\
\text { (SE) }\end{array}$ & $p$ & $d$ & $\begin{array}{l}\text { SLT present Mean } \\
\text { (SE) }\end{array}$ & $\begin{array}{l}\text { SLT absent Mean } \\
\text { (SE) }\end{array}$ & $p$ & $d$ \\
\hline No diagnosis & $52.4(0.78)$ & $52.7(0.09)$ & ns & 0.03 & $49.5(0.96)$ & $50.4(0.15)$ & ns & 0.09 \\
\hline Any SU, MI, or PD & $44.3(0.80)$ & $47.3(0.14)$ & $<0.001$ & 0.25 & $42.9(0.70)$ & $45.2(0.14)$ & $<0.01$ & 0.20 \\
\hline Any SU or MI & $43.9(0.84)$ & $47.1(0.16)$ & $<0.001$ & 0.26 & $42.6(0.74)$ & $45.0(0.15)$ & $<0.01$ & 0.21 \\
\hline Any MI or PD & $42.7(0.94)$ & $46.0(0.17)$ & $<0.01$ & 0.26 & $41.7(0.85)$ & $44.1(0.15)$ & $<0.01$ & 0.20 \\
\hline Any SU or PD & $44.2(0.84)$ & $47.1(0.17)$ & $<0.01$ & 0.24 & $42.9(0.73)$ & $45.1(0.16)$ & $<0.01$ & 0.19 \\
\hline Comorbid SU and MI & $40.0(1.34)$ & $43.7(0.33)$ & $<0.01$ & 0.29 & $39.2(1.25)$ & $41.9(0.29)$ & $<0.05$ & 0.23 \\
\hline Comorbid SU and PD & $43.1(1.30)$ & $44.2(0.34)$ & ns & 0.09 & $41.1(1.19)$ & $42.6(0.31)$ & ns & 0.13 \\
\hline Comorbid MI and PD & $38.7(1.34)$ & $41.6(0.26)$ & $<0.05$ & 0.21 & $38.3(1.19)$ & $40.2(0.25)$ & ns & 0.16 \\
\hline Comorbid SU and PD and MI & $38.2(1.63)$ & $41.5(0.45)$ & ns & 0.17 & $37.9(1.53)$ & $40.2(0.42)$ & ns & 0.19 \\
\hline Any MI & $40.0(1.03)$ & $45.2(0.19)$ & $<0.0001$ & 0.40 & $39.7(0.94)$ & $43.3(0.17)$ & $<0.001$ & 0.30 \\
\hline Schizophrenia/Psychosis & $31.3(3.45)$ & $36.8(0.99)$ & ns & 0.39 & $31.1(2.79)$ & $36.7(0.89$ & ns & 0.43 \\
\hline Any mood disorder & $38.7(1.24)$ & $43.0(0.28)$ & $<0.001$ & 0.32 & $38.2(1.21)$ & $41.3(0.24)$ & $<0.05$ & 0.25 \\
\hline Major depression & $39.1(1.28)$ & $43.3(0.28)$ & $<0.01$ & 0.32 & $39.0(1.10)$ & $41.7(0.25)$ & $<0.05$ & 0.22 \\
\hline Persistent depressive & $33.7(1.82)$ & $39.7(0.61)$ & $<0.01$ & 0.42 & $33.5(1.78)$ & $38.4(0.53)$ & $<0.05$ & 0.37 \\
\hline Bipolar 1 & $39.9(3.90)$ & $40.8(0.75)$ & ns & 0.07 & $36.2(4.34)$ & $38.8(0.65)$ & ns & 0.21 \\
\hline Any anxiety disorder & $37.5(1.24)$ & $44.6(0.25)$ & $<0.0001$ & 0.54 & $37.4(1.22)$ & $43.0(0.23)$ & $<0.0001$ & 0.45 \\
\hline Specific phobia & $38.6(2.47)$ & $45.9(0.36)$ & $<0.01$ & 0.57 & $38.9(1.89)$ & $44.5(0.30)$ & $<0.01$ & 0.47 \\
\hline Social anxiety & $34.5(2.59)$ & $41.7(0.53)$ & $<0.01$ & 0.52 & $35.3(2.73)$ & $41.3(0.48)$ & $<0.05$ & 0.47 \\
\hline Panic & $34.4(2.29)$ & $39.7(0.57)$ & $<0.05$ & 0.36 & $32.5(2.13)$ & $39.1(0.53)$ & $<0.01$ & 0.50 \\
\hline Agoraphobia & $32.1(3.04)$ & $39.4(0.68)$ & $<0.05$ & 0.52 & $34.4(2.19)$ & $38.0(0.68)$ & ns & 0.28 \\
\hline Generalized anxiety & $35.7(1.64)$ & $42.4(0.40)$ & $<0.001$ & 0.49 & $33.8(1.80)$ & $40.8(0.35)$ & $<0.001$ & 0.56 \\
\hline Posttraumatic stress & $34.9(1.81)$ & $41.6(0.42)$ & $<0.001$ & 0.48 & $35.6(1.85)$ & $40.1(0.35)$ & $<0.05$ & 0.34 \\
\hline Eating disorder ${ }^{a}$ & $36.4(3.78)$ & $44.1(0.75)$ & $<0.05$ & 0.60 & $34.6(3.50)$ & $43.4(0.79)$ & $<0.05$ & 0.70 \\
\hline Any substance use disorder & $45.1(0.92)$ & $48.7(0.19)$ & $<0.001$ & 0.32 & $43.3(0.84)$ & $46.4(0.19)$ & $<0.001$ & 0.29 \\
\hline Alcohol abuse & $45.6(0.99)$ & $49.3(0.17)$ & $<0.001$ & 0.34 & $44.0(0.79)$ & $47.0(0.19)$ & $<0.001$ & 0.28 \\
\hline Drug abuse & $43.9(1.27)$ & $44.6(0.49)$ & ns & 0.05 & $40.8(1.24)$ & $42.4(0.47)$ & ns & 0.13 \\
\hline Any personality disorder & $42.5(1.12)$ & $44.6(0.21)$ & ns & 0.16 & $41.3(1.00)$ & $43.0(0.20)$ & ns & 0.14 \\
\hline Borderline & $41.3(1.14)$ & $43.0(0.24)$ & ns & 0.12 & $40.2(1.04)$ & $41.5(0.25)$ & ns & 0.11 \\
\hline Antisocial & $43.5(1.53)$ & $46.3(0.38)$ & ns & 0.21 & $41.7(1.44)$ & $44.2(0.37)$ & ns & 0.20 \\
\hline Schizotypal & $41.4(1.70)$ & $42.5(0.33)$ & ns & 0.08 & $39.6(1.63)$ & $41.0(0.36)$ & ns & 0.11 \\
\hline
\end{tabular}

Note: All data weighted to adjust for nonresponse. Psychiatric categories not mutually exclusive. Lower scores indicate poorer perceived functioning. $2<$ Cohen $d<5$; Cohen $d \geq 5$. ${ }^{a}$ Includes bulimia and anorexia nervosa. 
Table 3. Lifetime incarceration (LI): effect size of functional impairment by lifetime diagnosis.

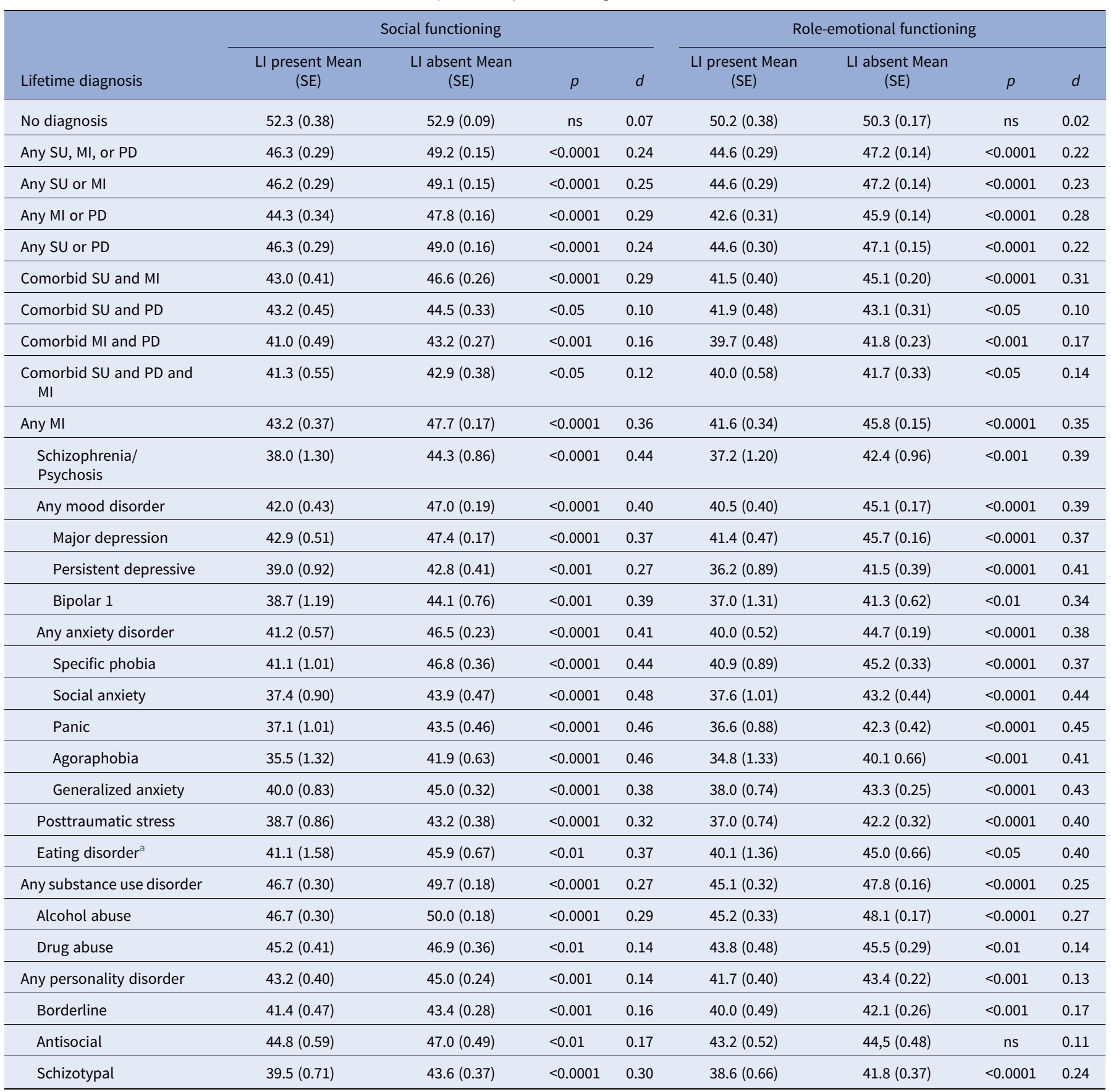

Note: All data weighted to adjust for nonresponse. Psychiatric categories not mutually exclusive. Lower scores indicate poorer perceived functioning. $2<$ Cohen $d<5$; Cohen $d \geq 5$. ancludes bulimia and anorexia nervosa.

(see Tables 3 and 4). Almost all forms of mental illness and alcohol abuse showed a small statistical effect in the direction of participants who described a legally significant outcome having greater impairment. The exception to this rule was role-emotional function and lifetime violence in schizophrenia/psychosis for which the effect size was moderate. There was no significant effect of social or role-emotional function in participants who did not have a mental disorder. Effect sizes were small or nonsignificant in drug abuse and the personality disorders.
In light of previous suggestions of a curvilinear relationship between function and violence in psychosis we divided role-emotional function scores of participants with schizophrenia/psychosis into quintiles and calculated the prevalence of serious trouble with the police or the law in each quintile. The numbers were smaller than for other diagnostic categories (see Table 1). With that caveat, we did not find evidence of a curvilinear relationship (prevalence of serious trouble in five centiles, lowest functioning to highest, 32.1, $25,17.9,14.3$, and $10.7 \%)$. 
Table 4. Lifetime violence (LV) to others: effect size of functional impairment by lifetime diagnosis.

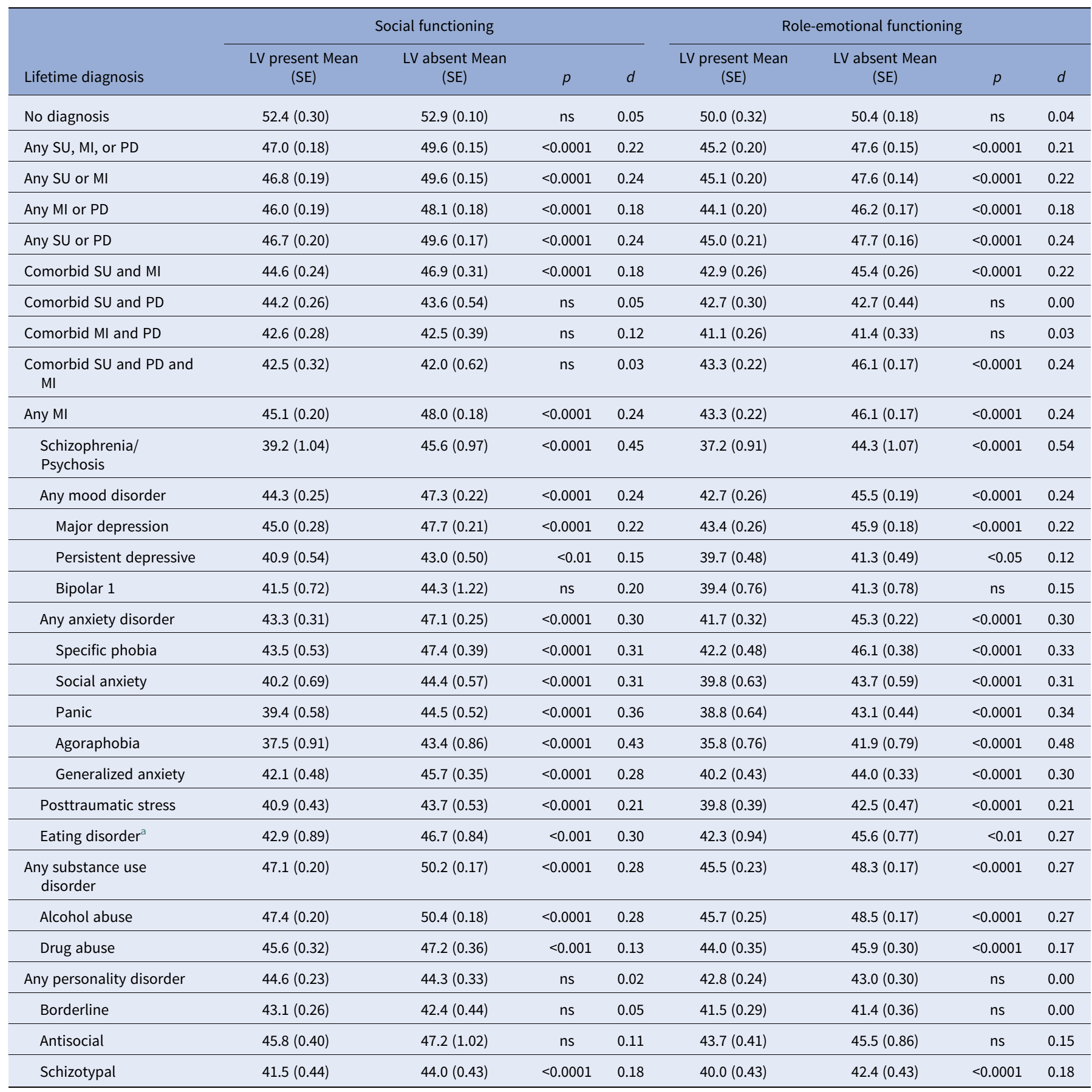

Note: All data weighted to adjust for nonresponse. Psychiatric categories not mutually exclusive. Lower scores indicate poorer perceived functioning. $2<$ Cohen $d<5$; Cohen $d \geq 5$.

ancludes bulimia and anorexia nervosa.

\section{Discussion}

In a large and representative sample of the US population, people with mental disorders who report recent and serious trouble with the police or law, lifetime incarceration or lifetime violence to others also report impaired social and role-emotional functioning as consequence of emotional problems compared with people with mental disorders who do not report these outcomes. The association is statistically significant, diagnosis dependent and of up to moderate effect size. People without a mental disorder do not show it.
Because the NESARC-III obtained data at a single interview, participants' functional impairment could have started before, during or after any of the three forms of legal involvement that we measured. This is particularly the case for the two lifetime measures (Tables 3 and 4). However, most of the diagnoses studied are either chronic or relapsing conditions that could be expected to be present for much of a participant's life and participants were asked to limit their descriptions of functional impairment to instances in which the impairment was the result of emotional problems. Especially for the 12-month measures, it is more likely 
than not that any functional impairment bore a temporal relationship to the outcome variable.

The association between legally significant outcomes and functional impairment is greatest for mental illness and smallest for the personality disorders. For substance use the picture is mixed. In alcohol use disorder, but not in drug use, function is significantly associated with all three outcomes. It is possible that the behavioral nexus between the possession and distribution of illegal drugs, on the one hand, and violence and legal involvement, on the other, generates a ceiling effect, an association between diagnosis, criminal behavior and legal trouble that is already too substantial to be increased by any additional functional impairment secondary to substance abuse.

Function is most strongly associated with legally significant outcomes in those diagnoses where the odds of the outcome are lowest. The two largest associations between impaired function and 12-month trouble with the police or law, for instance, are for specific phobias and eating disorders, diagnoses with the lowest rates of legal trouble (see Table 1). In drug abuse and the personality disorders, legally significant outcomes are more frequent but the statistical effects of function are either small or statistically insignificant. The contribution of impaired psychosocial function may be analogous to the contribution of mentally abnormal homicide to homicide rates internationally. The contribution of mentally abnormal homicide appears to be relatively constant in terms of numbers and is therefore smaller in percentage terms in jurisdictions where other risk factors are more common and homicide rates are higher [23].

The association between function and legally significant outcomes is larger for the 12-month data (Table 2) than for the lifetime data (Tables 3 and 4). Most mental conditions vary in severity over their course. Even if social and role-emotional function was exerting a direct effect on the legal involvement, that effect could be expected to be less evident when function is measured after, perhaps long after, behavior. Some people who were functionally impaired when they acted violently, for instance, will have improved by the time their function was assessed, reducing the statistical association.

What do these findings suggest about the mechanisms linking mental disorder to legal trouble, incarceration and violence? First, the variation in the size of the association across diagnoses suggests that multiple mechanisms are involved. Second, studies of delusions in patients who have recently been discharged from the hospital [24] and studies of court-involved people who describe symptoms and signs of serious mental illness [25] both suggest that it is relatively unusual for symptoms and signs of illness to cause violence directly. Our data are more consistent with the conclusions suggested by these studies, that causal links are multiple and indirect, for instance operating through the damage that mental illness can cause to the family and social networks that would otherwise protect against incarceration, violence and other forms of legal involvement.

These data make some alternative explanations for the associations between function and legally significant outcomes less likely. It is possible, for instance, that the experience of having serious trouble with the police or law colored participants' perception of their own levels of function. But had this been the case we would have expected the association between function and legally significant outcomes to be evident in nondisordered participants also, albeit to a lesser degree given that some of those participants will not have experienced the "emotional problems" that SF-12v12 items ask about. It is also possible that the change in function was a consequence, rather than a cause, of being in trouble with the police or law [26]. Again, however, had this been the case we would have expected reduced function, albeit perhaps to a lesser degree, in nondisordered participants.

These findings warrant several caveats. First, NESARC-III data derive exclusively from interviews with participants. We were not able to augment the data using collateral sources, for instance regarding incarceration and legal contacts. Second, the "schizophrenia/psychosis" variable was generated from a single NESARC item and not from the AUDADIS interview. While the approach we used was the same as that used by other studies using this sample [27], an interview-derived measure might have generated different results. Third, while the measures that generated our social function and role-emotional function variables have been extensively tested and widely used, we were not able to use collateral data to supplement and corroborate participants' self-report. Fourth, and particularly for disadvantaged groups, engaging in a criminal act is not a necessary pre-requisite for legal involvement. People with mental disorders are also more likely than others to be apprehended when they do commit a criminal offense [28].

Most people who suffer from mental disorders have no history of behaving violently. But understanding the reasons for the exceptions to this rule remains of importance to patients, to potential victims (most often family members) and to mental health services. Future studies could advance the field by addressing the caveats we list here. The larger task, however, is to establish the nature of the links, described here as likely to be indirect and multiple, between social and role-emotional functioning and violence. Studies that can do that are likely to require detailed measures of legal involvement, robust measurement of causal factors and, ideally, longitudinal designs.

Data Availability Statement. Data are available at https://www.niaaa.nih. gov/research/nesarc-iii.

Author Contributions. Conceptualization: A.B., K.E.M., S.A.M.; Data curation: A.B., K.E.M., B.P., S.A.M.; Formal analysis: A.B., K.E.M.; Methodology: A.B., K.E.M., B.P., S.A.M.; Project administration: A.B., S.A.M.; Writingoriginal draft: A.B., K.E.M., S.A.M.; Writing-review and editing: A.B., K.E.M., B.P., S.A.M.

Conflict of Interest. The authors declare none.

\section{References}

1. Corrigan P, Watson A. Findings from the National Comorbidity Survey on the frequency of violent behavior in individuals with psychiatric disorders. Psychiatry Res. 2005;136:153-62.

2. Hodgins S. Mental disorder, intellectual deficiency, and crime: evidence form a birth cohort. Arch Gen Psychiatry. 1992;49:476-83.

3. Arseneault L, Moffitt T, Caspi A, Taylor PJ, Silva PA. Mental disorder and violence in a total birth cohort. Arch Gen Psychiatry. 2000;57:979-86.

4. Swanson J, Holzer C, Ganju V, Jono RT. Violence and psychiatric disorder in the community: evidence from the epidemiologic catchment area surveys. Hosp Community Psychiatry. 1990;41:761-70.

5. Elbogen E, Johnson S. The intricate link between violence and mental disorder. Results from the National Epidemiologic Survey on alcohol and related conditions. Arch Gen Psychiatry. 2009;66:152-61.

6. Van Dorn R, Volavka J, Johnson N. Mental disorder and violence: is there a relationship beyond substance use? Soc Psychiatry Psychiatr Epidemiol. 2012;47:487-503.

7. Coid J, Ullrich S, Bebbington P, Fazel S, Keers R. Paranoid ideation and violence: meta-analysis of individual subject data of 7 population surveys. Schizophrenia Bull. 2016;42:907-15. 
8. Ustun B, Kennedy C. "What is functional impairment"? Disentangling disability from clinical significance. World Psychiatry. 2009;8:82-5.

9. American Psychiatric Association. Diagnostic and statistical manual of mental disorders, DSM-V. 5th ed. Arlington, VA: American Psychiatric Association; 2013.

10. World Health Organization. ICD-10: International statistical classification of diseases and related health problems. 10th rev., 2nd ed. Geneva: World Health Organization; 2004.

11. Swanson J, Swartz M, Essock S, Osher FC, Wagner HR, Goodman LA, et al. The social-environmental context of violent behavior in persons treated for severe mental illness. Am J Public Health. 2002;92:1523-31.

12. Witt K, Van Dorn R, Fazel S. Risk factors for violence in psychosis: systematic review and meta-regression analysis of 110 studies. PLoS One. 2013;8:e55942.

13. Swanson J, Swartz M, Estroff S, Borum R, Wagner R, Hiday V. Psychiatric impairment, social contact, and violent behavior: evidence from a study of outpatient-committed persons with severe mental disorder. Soc Psychiatry Psychiatr Epidemiol. 1998;33:S86-94.

14. Swanson J, Estroff S, Swartz M, Borum R, Lachicotte W, Zimmer C, et al. Violence and severe mental disorder in clinical and community populations: the effects of psychotic symptoms, comorbidity, and lack of treatment. Psychiatry. 1997;60:1-22.

15. Grant B, Chu A, Sigman R. Source and accuracy statement: National Epidemiologic Survey on alcohol and related conditions-III (NESARCIII). Rockville, MD: National Institute on Alcohol Abuse and Alcoholism; 2014.

16. Grant B, Saha T, Ruan WJ, Goldstein RB, Chou SP, Jung J, et al. Epidemiology of DSM 5 drug use disorder: results from the National Epidemiologic Survey of alcohol and related conditions (NESARC) - III. JAMA Psychiatry. 2016;73:39-47.

17. Grant B, Goldstein R, Smith S, Jung J, Zhang H, Chou SP, et al. The alcohol use disorder and associated disabilities interview Schedule-5 (AUDADIS-
5): reliability of substance use and psychiatric disorder modules in a general population sample. Drug Alcohol Depend. 2015;148:27-33.

18. Harford T, Chen C, Kerridge B. Self- and other-directed forms of violence and their relationship with lifetime DSM-5 psychiatric disorders: results from the National Epidemiologic Survey on alcohol related conditions-III (NESARC-III). Psychiatry Res. 2018;262:384-92.

19. Gandek B, Ware J, Aaronson N, Alonso J, Apolone G, Bjorner J, et al. Tests of data quality, scaling assumptions, and reliability of the SF-36 in eleven countries: results from the IQOLA project. J Clin Epidemiol. 1998;51: 1149-58.

20. McHorney C, Ware J, Lu R. The MOS 36-item short-form health survey (SF-36): III. Tests of data quality, scaling assumptions, and reliability across diverse patient groups. Med Care. 1994;32:40-66.

21. Ware J, Sherbourne D. The MOS 36-item short-form health survey (SF-36). Med Care. 1992;30:473-83.

22. SAS Institute Inc 2013. SAS/ACCESS ${ }^{\bullet} 9.4$ Interface to ADABAS: Reference. Cary, NC: SAS Institute Inc.

23. Coid J. The epidemiology of abnormal homicide and murder followed by suicide. Psychol Med. 1983;13:855-60.

24. Appelbaum P, Robbins P, Monahan J. Violence and delusions: data from the MacArthur violence risk assessment study. Am J Psychiatry. 2000;157:566-72.

25. Peterson J, Skeem J, Kennealy P, Bray B, Zvonkovic A. How often and how consistently do symptoms directly precede criminal behavior among offenders with mental illness. Law Hum Behav. 2014;38:439-49.

26. Schnittker J, Massoglia M, Uggen C. Out and down: incarceration and psychiatric disorders. J Health Soc Behav. 2012;53:448-64.

27. Moore K, Oberleitner L, Zonana H, Buchanan AW, Pittman BP, Verplaetse TL, et al. Psychiatric disorders and crime in the US population: results from the National Epidemiologic Survey on alcohol and related conditions wave III. J Clin Psychiatry. 2019;80(2):18m12317.

28. Robertson G. Arrest patterns among mentally disordered offenders. Br J Psychiatry. 1988;153:313-6. 